\section{Pseudomonas aeruginosa bacteraemia in two UK district hospitals}

\author{
David A. Enoch, ${ }^{1}$ Julie Kuzhively, ${ }^{2}$ \\ Andrew Sismey, ${ }^{1}$ Alina Grynik, ${ }^{3}$ \\ Johannis Andreas Karas, \\ ${ }^{1}$ Clinical Microbiology and Public Health \\ Laboratory, Peterborough and Stamford \\ Hospitals NHS Foundation Trust, \\ Peterborough City Hospital, Bretton \\ Gate, Peterborough; ' 2 Department of \\ Medicine, Peterborough and Stamford \\ Hospitals NHS Foundation Trust, \\ Peterborough City Hospital, Bretton \\ Gate, Peterborough; ${ }^{3}$ Infection Control \\ Department, Hinchingbrooke Hospital, \\ Huntingdon; ${ }^{4} \mathrm{Clinical}$ Microbiology and \\ Public Health Laboratory, Health \\ Protection Agency, Addenbrookes \\ Hospital, Cambridge, UK
}

\section{Abstract}

Pseudomonas aeruginosa bacteraemia is associated with significant morbidity and mortality. We retrospectively studied the epidemiology of bacteraemia due to $P$. aeruginosa in two UK district hospitals so as to determine prevention strategies and assess the efficacy and compliance with local hospital antibiotic guidelines. Eighty six episodes occurred in 85 patients over the 3 year period. There was a year on year increase in bacteraemias, due predominantly to an increased proportion of community-onset episodes. Urinary catheterisation was a significant risk factor, along with anaemia, renal disease, malignancy and diabetes. The antibiotic guidelines were adequate for $92.8 \%$ of episodes but only $73.8 \%$ of patients received adequate therapy. Failure to follow the guidelines was principally due to unwillingness to use gentamicin due to concerns about nephrotoxicity. The antibiotic guidelines may need reviewing to accommodate this problem and further work is required to address urinary catheter care in both the hospital and community. Pseudomonas aeruginosa should be considered a significant pathogen when patients are admitted with features of sepsis.

\section{Introduction}

Pseudomonas aeruginosa bacteraemia is associated with significant morbidity and mortality rates ranging from $20-38 \% .{ }^{1}$ Factors that are associated with increased mortality include antibiotic resistance and delays in appropriate antibiotic therapy. ${ }^{1-5}$

Risk factors for bacteraemia previously identified from a population-based study include male sex, increasing age, haemodialysis, solid organ transplant, cancer, diabetes and HIV infection. ${ }^{6}$ However, very few studies have been performed in district hospitals. ${ }^{7} \mathrm{We}$ sought to describe the epidemiology of bacteraemia due to $P$. aeruginosa in two UK district hospitals so as to determine prevention strategies and assess the efficacy and compliance with local hospital antibiotic guidelines.

\section{Materials and Methods}

\section{Setting}

Peterborough and Stamford Hospitals National Health Service (NHS) Foundation Trust and Hinchingbrooke Hospital NHS Trust (UK) are district general hospitals with 600 and 260 beds respectively including acute medical, surgical (general and orthopaedic), elderly care, women's services and intensive care. Together, they cover a predominantly semirural population of 600,000 people in Cambridgeshire, England with approximately 110,000 admissions per year.

\section{Study design and patients}

A retrospective observational study of all Pseudomonas aeruginosa bacteraemias occurring in all patients for the 3 years commencing July $1^{\text {st }} 2008$ was performed. The decision to take blood cultures was at the discretion of the clinician attending the patient. All bacteraemias were reviewed by a Consultant Microbiologist on receipt of the initial result and antibiotics changed to reflect the guidelines if non-compliant. The empirical treatment was likely to be modified at this point, or the following day, when the susceptibility results were available. Patients therefore rarely received inadequate therapy for more than 48 hours. Approval for the study was obtained from the Trust's audit committees.

\section{Variables}

Demographic data, clinical details and the antibiotic history were obtained from laboratory and microbiology records and medical, nursing and pharmacy notes as well as the patient administration system. Data obtained included age, gender, number of hospitalisations in the preceding year (including other hospitals), co morbidities (leg ulcers, open wounds, cardiac disease, intravenous drug use, alcohol abuse, renal disease (chronic kidney disease stage $\geq 2$ or raised serum creatinine $>120 \mu \mathrm{mol} / \mathrm{L}$ ), neurological disorder, diabetes, anaemia (haemoglobin <115 g/L), malignancy, liver disease,
Correspondence: Johannis Andreas Karas, Clinical Microbiology and Public Health Laboratory, Health Protection Agency, Addenbrookes Hospital, Cambridge, CB2 2QW, UK.

Tel. +44.01223.216818 - Fax: +44.01223.242775

E-mail: johannisandreas.karas@addenbrookes.nhs.uk

Key words: Pseudomonas aeruginosa, bacteraemia, community onset, adequacy of antibiotics.

Acknowledgements: the authors would thank the laboratory staff on all sites in assisting with this project. Clinical audit departments at Peterborough City Hospital and Hinchingbrooke Hospital.

Contributions: JAK and DAE conceived and planned the project; all authors contributed to data collection, analysis and writing up the report.

Conflict of interests: the authors declare no potential conflict of interests.

Received for publication: 4 November 2011.

Revision received: 2 April 2012

Accepted for publication: 4 April 2012.

This work is licensed under a Creative Commons Attribution NonCommercial 3.0 License (CC BYNC 3.0).

(C) Copyright D.A. Enochet al., 2013

Licensee PAGEPress, Italy

Infectious Disease Reports 2013; 5:e4

doi:10.4081/idr.2013.e4

immunosuppression [receipt of cytotoxic chemotherapy, immunosuppressant or prednisolone (30 mg/day or equivalent)], co-morbidity score (Charlson comorbidity index), antibiotic history post-bacteraemia, central venous catheter usage, urinary catheter usage, primary outcome data (death at one week and 30 days) and secondary outcome data [requirement for intensive care unit (ICU), requirement for surgery and recurrence].

\section{Definitions}

Bacteraemias were counted as one episode if they occurred within 14 days of a previous result (with the same organism and antibiogram). Subsequent episodes occurring after this time were considered new episodes and considered to be recurrences (with the same organism and antibiogram). Blood cultures growing more than one organism were defined as mixed. Community-onset (C) bacteraemia was defined as that occurring within 48 hours of admission if the patient was not transferred from another hospital, whereas hospital-onset (H) bacteraemia was after this period. Healthcare-associated (HCAI) bacteraemia was defined as blood cultures taken within 48 hours of hospital admission from a patient who had been admitted to hospital in the preceding 
year or from a nursing home. Shock was defined as sepsis associated with evidence of organ hypoperfusion and a systolic blood pressure of $<90$ or $>30 \mathrm{mmHg}$ less than the baseline that did not respond to fluid treatment and required vasoactive drug therapy. Empirical therapy was considered adequate if the agent(s) initially given had in vitro activity against the organism subsequently identified. Length of stay was defined as days after blood culture taken until discharge.

The hospital antibiotic guidelines (limited to relevant indications only) recommend the combination of gentamicin and co-amoxiclav (or gentamicin and ciprofloxacin if penicillin allergic) for empiric treatment of severe urinary tract infection or pyelonephritis, biliary and abdominal sepsis and sepsis of unknown source. Amoxicillin is recommended for early onset hospital-acquired pneumonia (onset within 4 days of admission), co-amoxiclav for late onset and piperacillin-tazobactam for ventilator associated pneumonia). Piperacillintazobactam (or ciprofloxacin) and gentamicin is the empirical treatment of choice for neutropenic sepsis. Co-amoxiclav is recommended for the treatment of moderate diabetic foot infections with piperacillin-tazobactam reserved for severe diabetic foot infections. Vancomycin and gentamicin is recommended for suspected line infection, while meropenem is recommended in patients with previously colonised or infected with extended spectrum beta-lactamase (ESBL) producing organisms.

\section{Microbiology}

Microbiology data was obtained from the laboratory information systems. All blood cultures were processed using the BacT/Alert 3D system (bioMérieux UK Ltd, Basingstoke, UK). Organisms were identified to species level by API20E or API20NE (bioMérieux UK Ltd, Basingstoke, UK). Routine antibiotic susceptibility testing was performed according to British Society for Antimicrobial Chemotherapy (BSAC) methodology. Antibiotics tested included amoxicillin, co-amoxiclav, ciprofloxacin, trimethoprim, gentamicin, cefuroxime, ceftazidime, ceftriaxone, piperacillin-tazobactam and meropenem.

\section{Statistical analysis}

Fisher's exact test was used for statistical analysis of categorical data.

\section{Results}

Ninety five bacteraemias (88 episodes) occurred in 87 patients over the three year period. Information was available for 86 episodes occurring in 85 patients. Three patients had a recurrence. Fifty three (60\%) were male and ages ranged from 0-95 (median 73, interquartile range $=60-84)$. Onset was mostly HCAI (52 episodes) followed by hospital (23), community (10) and unknown (1) (Table 1). We were unable to find any healthcare contact for the 10 patients that had community infections. Onset changed over the years, with an increased proportion due to HCAI and community onset episodes (from 2008/9 to 2010/11) which was highly statistically significant $(\mathrm{P}<0.0002)$. Most patients were medical, followed by oncology and surgery and three patients were present on ICU this included one community case that was admitted directly. Of hospital cases $26 \%$ were surgical cases compared to 0 and $3.8 \%$ of community and HCAI cases. The urinary tract was the most frequent source (33) followed by the respiratory tract (20), unknown (11), skin and soft tissue (9), bowel (7), central venous catheter (3) and biliary tract (2).

The most frequent risk factor was presence of a urinary catheter (42 episodes; the source in 24 of these cases). Eighteen of these were community onset (all HCAI) and six were hospital onset. Malignancy (39), dependent functional state (34), renal disease (33), immunosuppression (33) and diabetes (23) were other risk factors. Fifteen patients had a central venous catheter. Ten patients had a Charlson co-morbidity score of 0 ; however, five of these had urinary catheters in-situ. Fifteen episodes (17.4\%) had an absolute neutrophil count of less than $1.5 \times 10^{9} / \mathrm{L}$ and four had an absolute neutrophil count of $\leq 0.1 \times 10^{9} / \mathrm{L}$. Anaemia was present in 46 (53\%) episodes and in 15 episodes the patient had received care from a district nurse. One patient had underlying connective tissue disease and there were no HIV sero-positive patients or intravenous drug users.

The local hospital antibiotic guidelines were adequate for $92.8 \%$ of episodes and the diagnosis was correct for $96.3 \%$ of episodes. Only $73.8 \%$ of patients received adequate therapy. The guidelines were followed in $81.2 \%$ of episodes. Failure to follow the guidelines was failure to prescribe gentamicin (10 episodes), not treating the bacteraemia (4), failure to treat diabetic ulcers (3), receipt of targeted therapy (2), and misdiagnosis (1).

Seventy one episodes (81\%) had a temperature $\geq 38^{\circ} \mathrm{C}$ and 28 of 78 (26.9\%) had a blood pressure of $<90 \mathrm{mmHg}$. Twelve patients required admission to ICU and 10 required surgery. The mortality at 7 days and 28 days was $25.9 \%$, and $32.9 \%$ respectively.

Fifteen episodes were mixed (viridans type streptococci $\times 5$, E. faecalis $\times 5$, coagulase negative staphylococci $\times 3$, Citrobacter koseri $\times 2, E$. coli $\times 2$, and one Klebsiella pneumoniae). All isolates were gentamicin and piperacillintazobactam susceptible. Eight isolates were resistant to ciprofloxacin, three to meropenem and one to ceftazidime.

\section{Discussion}

Our study confirms previous studies of risk factors by finding male sex, advanced age, cancer and diabetes to be important risk factors. ${ }^{6}$ This study also found HIV infection and haemodialysis to be risk factors. A male predominance has been noted in a number of other studies. ${ }^{1,8}$

Though the numbers are small, there was an increase in numbers over the study period, from 24 episodes per year (2008-9) to 36 episodes in the final year. This reflected a rise in community and HCAI onset cases (from 11 to 31 which was statistically significant).

A number of studies have reported on risk factors for community-onset bacteraemias. We detected ten patients without hospital or nursing home contact that we considered true community acquired cases and only one of these had a urinary catheter which was not the source of infection. Three cases had a urinary tract source, three had a respiratory source and two had a wound infection source. No source was determined for two patients. The majority of community-onset bacteraemias have prior hospitalisation or were admitted from a nursing home (i.e. HCAI). ${ }^{9,10}$ Other risk factors for community onset previously identified include neutropenia, ${ }^{9,11}$ septic shock and presence of a central venous catheter and / or a urinary catheter. ${ }^{9,10}$

Our study found that the urinary tract was the most frequent source $(43.5 \%$; with the majority being catheter related), followed by the respiratory tract and unknown. The biliary tract was rarely implicated. The urinary tract was the most frequent source in another study, ${ }^{2}$ but others have found the most common source to be unknown, $, 3,4$ the respiratory tract or the biliary tract. ${ }^{6,10}$ Invasive devices (urinary catheters and central venous catheters) have been frequently implicated previously. ${ }^{10,11}$ All patients had a past medical history. One study suggested only two of 39 patients had no past medical history. ${ }^{8}$ Of note is the higher proportion of hospital onset cases that were related to surgery, this is also likely to reflect higher rates of urinary catheterisation in this group.

We noticed a rise in the number of urinary catheter related bacteraemias, particularly of HCAI-onset. This may reflect a change in practice with increased attention to urinary catheter care in the hospital but may also reflect earlier discharge from hospital.

Twenty two (26.2\%) of our patients had a delay in adequate therapy; this was always less than 48 hours. The most frequent reason for inadequate therapy was failure to give gentamicin due to concerns about nephrotoxicity. We have found this previously. ${ }^{12}$ Despite audit and education, we found no improvement in pre- 
scribing habit over the 3 -year period. Interestingly, patients with hospital-onset bacteraemia were less likely to receive adequate therapy than community and HCAI-onset bacteraemias. One concern may be the high rate of renal disease and the subsequent reluctance to prescribe aminoglycosides; $39 \%$ of patients in our study had renal disease. Our results are comparable to some studies and better than others. Around $50 \%$ of bacteraemias were initially treated inadequately in several studies, ${ }^{1,5,13}$ while another study found one $24.6 \%$ were inadequately treated. ${ }^{4}$ Micek and colleagues found that waiting more than 52 hours was associated with increased mortality. ${ }^{4}$ Studies of community onset bacteraemias have found rates of inadequate therapy between $37-61 \% .8,10,11,14$ Our mortality at 30 days was $32.9 \%$. This is within the range described by others (21-39\%). . $^{3-6,8,10,11}$

Our study has low levels of antimicrobial resistance, which may reflect the district hospital setting. No resistance to piperacillintazobactam or gentamicin, $3.5 \%$ to meropenem and $1.2 \%$ to ceftazidime compares favourably with the UK rates of resistance of $3.1 \%, 8.1 \%$ and $5.5 \%$ to those agents respectively as reported in the 2009 European surveillance report. ${ }^{15}$ Our rate of fluoroquinolone resistance of $9 \%$ was at the upper limit of the rate in the UK. Approximately $18 \%$ of the bacteraemias were mixed, which has been described previously. ${ }^{2}$

\section{Conclusions}

This study has a number of limitations. The retrospective nature means some data is missing and the numbers are small. No molecular studies were used to categorise samples fur-

Table 1. Table of results by onset category.

\begin{tabular}{|c|c|c|c|c|c|}
\hline & 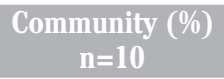 & $\begin{array}{c}\text { Hospital (\%) } \\
n=23\end{array}$ & $\begin{array}{c}\text { HCAI (\%) } \\
n=52\end{array}$ & $\begin{array}{c}\text { Unknown } \\
n=3\end{array}$ & $\begin{array}{c}\text { Total (\%) } \\
n=85\end{array}$ \\
\hline $\begin{array}{l}\text { Onset (episodes) } \\
2008-09 \\
2009-10 \\
2010-2011\end{array}$ & $\begin{array}{l}1(10) \\
2(20) \\
7(70)\end{array}$ & $\begin{array}{l}13(57) \\
5(22) \\
5(22)\end{array}$ & $\begin{array}{l}10(19) \\
18(35) \\
24(46)\end{array}$ & $\begin{array}{l}0 \\
1 \\
0\end{array}$ & $\begin{array}{l}24(28) \\
26(30) \\
36(42)\end{array}$ \\
\hline $\begin{array}{l}\text { Specialty } \\
\text { Medicine } \\
\text { Cancer } \\
\text { Surgery } \\
\text { ICU } \\
\text { Orthopaedics } \\
\text { Paediatrics } \\
\end{array}$ & $\begin{array}{c}9(90) \\
0 \\
0 \\
1(10) \\
0 \\
0\end{array}$ & $\begin{array}{l}11(48) \\
3(13) \\
6(26) \\
2(9) \\
1(4) \\
0\end{array}$ & $\begin{array}{c}37(71) \\
12(23) \\
2(4) \\
0 \\
0 \\
1(2)\end{array}$ & $\begin{array}{l}2 \\
0 \\
0 \\
0 \\
0 \\
0\end{array}$ & $\begin{array}{c}59(69) \\
15(17) \\
8(9) \\
3(4) \\
1(1) \\
1(1)\end{array}$ \\
\hline $\begin{array}{l}\text { Source of infection } \\
\text { Urine } \\
\text { Catheter-associated } \\
\text { Pneumonia } \\
\text { Biliary tract } \\
\text { Bowel } \\
\text { Other } \\
\text { Wound } \\
\text { Unknown } \\
\text { Joint } \\
\text { Line }\end{array}$ & $\begin{array}{c}3(30) \\
0 \\
3(30) \\
0 \\
1(10) \\
\\
2(20) \\
1(10) \\
0 \\
0\end{array}$ & $\begin{array}{c}6(26) \\
6(100) \\
7(30) \\
1(4) \\
2(9) \\
4(17) \\
1(4) \\
1(4) \\
1(4)\end{array}$ & $\begin{array}{c}24(46) \\
18(75) \\
10(19) \\
1(2) \\
4(8) \\
\\
3(6) \\
9(17) \\
0 \\
2(4)\end{array}$ & $\begin{array}{l}0 \\
0 \\
0 \\
0 \\
0 \\
\\
0 \\
0 \\
0 \\
0\end{array}$ & $\begin{array}{c}33(43) \\
24(80) \\
20(26) \\
2(2) \\
7(8) \\
9(11) \\
12(14) \\
1(1) \\
3(4)\end{array}$ \\
\hline $\begin{array}{l}\text { Risk factors } \\
\text { Urinary catheter } \\
\text { Immunosuppression } \\
\text { Renal disease } \\
\text { Neurological disease } \\
\text { Central line } \\
\text { Neutropenia } \\
\text { Chronic wound - ulcer }\end{array}$ & $\begin{array}{l}1(10) \\
1(10) \\
5(50) \\
2(20) \\
0 \\
1(10) \\
1(10)\end{array}$ & $\begin{array}{l}18(78) \\
8(35) \\
10(44) \\
4(17) \\
5(22) \\
3(13) \\
7(30)\end{array}$ & $\begin{array}{l}21(40) \\
24(46) \\
18(35) \\
11(21) \\
10(19) \\
11(21) \\
5(10)\end{array}$ & $\begin{array}{l}2 \\
0 \\
0 \\
1 \\
0 \\
0 \\
0\end{array}$ & $\begin{array}{l}42(49) \\
33(38) \\
33(38) \\
18(21) \\
15(18) \\
15(18) \\
13(15)\end{array}$ \\
\hline $\begin{array}{l}\text { Co-morbidities } \\
\text { Malignant solid tumour } \\
\text { Diabetes } \\
\text { Cardiac disease } \\
\text { Myocardial infarction } \\
\text { COPD } \\
\text { Leukaemia / lymphoma } \\
\text { Liver disease } \\
\text { CVA } \\
\text { Hemiplegia } \\
\text { PVD } \\
\text { Dementia } \\
\text { Shock }\end{array}$ & $\begin{array}{l}1(10) \\
2(20) \\
3(30) \\
3(30) \\
2(20) \\
1(10) \\
1(10) \\
1(20) \\
0 \\
0 \\
0 \\
2 / 9(22)\end{array}$ & $\begin{array}{l}7(30) \\
5(22) \\
4(17) \\
6(26) \\
6(26) \\
2(9) \\
5(22) \\
2(9) \\
2(9) \\
5(21) \\
1(4) \\
5 / 21(24)\end{array}$ & $\begin{array}{c}20(39) \\
16(31) \\
11(21) \\
9(17) \\
8(15) \\
8(15) \\
4(8) \\
5(10) \\
5(10) \\
2(4) \\
4(8) \\
21 / 45(47)\end{array}$ & $\begin{array}{l}0 \\
0 \\
0 \\
0 \\
0 \\
0 \\
0 \\
0 \\
0 \\
0 \\
0\end{array}$ & $\begin{array}{l}28(33) \\
23(27) \\
18(21) \\
18(21) \\
16(19) \\
11(13) \\
10(12) \\
8(9) \\
7(8) \\
7(8) \\
5(6) \\
28 / 75(37.3)\end{array}$ \\
\hline
\end{tabular}

HCAI, healthcare-associated; CVC, central venous catheter; ICU, intensive care unit; PVD, peripheral vascular disease; CTD, connective tissue disease; COPD, chronic obstructive pulmonary disease; CVA, cerebrovascular accident. 
ther, in order to determine cross resistance or resistance mechanisms. Nevertheless, we believe this study adds further information to the epidemiology of a significant pathogen in the district hospital setting.

We have highlighted the role of urinary catheters as a significant risk for Pseudomonas aeruginosa bacteraemia; concerted and coordinated efforts are required both in the hospital and community to tackle this. The high rate of renal disease in this group of patients makes clinicians reluctant to use aminoglycosides and the local guidelines may need to be reviewed to accommodate this problem. The majority of cases were community onset (though with prior hospital contact). The results of this study suggest that Pseudomonas aeruginosa, though not a common finding in district hospitals, should still be considered a significant pathogen when patients are admitted with features of sepsis.

\section{References}

1. Suárez C, Peña C, Gavaldà L, et al. Influence of carbapenem resistance on mortality and the dynamics of mortality in Pseudomonas aeruginosa bloodstream infection. Int J Infect Dis 2010;14 Suppl 3:e73-8.

2. Suárez C, Peña C, Tubau F, et al. Clinical impact of imipenem-resistant Pseudomonas aeruginosa bloodstream infections. J Infect 2009;58:285-90.

3. Kang CI, Kim SH, Kim HB, et al. Pseudomonas aeruginosa bacteremia: risk factors for mortality and influence of delayed receipt of effective antimicrobial therapy on clinical outcome. Clin Infect Dis 2003;7:745-51.

4. Micek ST, Lloyd AE, Ritchie DJ, et al. Pseudomonas aeruginosa bloodstream infection: importance of appropriate initial antimicrobial treatment. Antimicrob Agents Chemother 2005;49:1306-11.

5. Lodise TP Jr, Patel N, Kwa A, et al. Predictors of 30-day mortality among patients with Pseudomonas aeruginosa bloodstream infections: impact of delayed appropriate antibiotic selection. Antimicrob Agents Chemother 2007 51:3510-5.

6 . Parkins MD, Gregson DB, Pitout JD, et al. Population-based study of the epidemiology and the risk factors for Pseudomonas aeruginosa bloodstream infection. Infection 2010;38:25-32.

7. Harris DM, Gray PB. Pseudomonas aeruginosa and the general hospital: a six-year survey. J Hyg (Lond) 1974;73:249-54.

8. Cheong HS, Kang CI, Wi YM, et al. Inappropriate initial antimicrobial therapy as a risk factor for mortality in patients with community-onset Pseudomonas aeruginosa bacteraemia. Eur J Clin Microbiol Infect Dis 2008;27:1219-25.
9. Kang CI, Kim SH, Park WB, et al. Clinical features and outcome of patients with community-acquired Pseudomonas aeruginosa bacteraemia. Clin Microbiol Infect 2008;11:415-8.

10. Schechner V, Nobre V, Kaye KS, et al. Gram-negative bacteremia upon hospital admission: when should Pseudomonas aeruginosa be suspected? Clin Infect Dis 2009;48:580-6.

11. Cheong HS, Kang CI, Wi YM, et al. Clinical significance and predictors of communityonset Pseudomonas aeruginosa bacteremia. Am J Med 2008;121:709-14.

12. Enoch DA, Phillimore N, Mlangeni DA, et al. Outcome for Gram-negative bacteraemia when following restrictive empirical antibiotic guidelines. QJM 2011;104: 411-9.

13. Gellen-Dautremer J, Bert F, Panhard X, et al. Physicians fail to consider Pseudomonas aeruginosa as a potential pathogen in medicine patients with bacteremia. $\mathrm{J}$ Infect 2011;63:99-101.

14. Huang YC, Lin TY, Wang CH. Communityacquired Pseudomonas aeruginosa sepsis in previously healthy infants and children: analysis of forty-three episodes. Pediatr Infect Dis J 2002;21:1049-52.

15. European Centre for Disease Prevention and Control. Antimicrobial resistance surveillance in Europe 2009. Annual Report of the European Antimicrobial Resistance Surveillance Network (EARS-Net). 